\title{
Optimal design of watt six-bar transmission mechanism for morphing trailing edge
}

\author{
Jin Zhou ${ }^{1,2}$, and Shiwei Zhao ${ }^{1, *}$ \\ ${ }^{1}$ School of Aeronautic Science and Engineering, Beihang University, Beijing 100191, China \\ ${ }^{2}$ Chinese Aeronautical Establishment, Beijing 100012, China
}

\begin{abstract}
Keywords: morphing trailing edge, watt six-bar transmission mechanism, optimal design.

Abstract. The morphing trailing edge could realize a continuous smooth deformation compared with conventional trailing edge, which effectively improves the aerodynamic performance. In this paper, a multi-step optimization design of watt six-bar transmission mechanism for morphing trailing edge is proposed. In the first optimization stage, the most effective aerodynamic shape and bar position in the middle of the morphing trailing edge is determined. In the second optimization stage, a watt six link transmission mechanism is proposed by using genetic algorithm to match the optimal shape from the first optimization stage. Result shows that the optimal design could achieve the determined aerodynamic shape in the first optimization stage perfectly.
\end{abstract}

\section{Introduction}

The morphing trailing edge could realize a continuous smooth deformation compared with conventional trailing edge, which effectively improves aerodynamic performance, expands flight envelope, reduces fuel consumption and decreases noise [1-3].

At present, many kinds of morphing trailing edge based on flexible materials and structures have been studied. Yokozeki et al. [4,5] proposed a variable geometry morphing airfoil using corrugated structure. Woods et al. [6-8] proposed a fish-bone-active-camber concept which consists of a thin chordwise bending beam spine with stringers branching off to connect it to a pretensioned elastomeric matrix composite (EMC) skin surface. Zhang et al.[9] designed a morphing wing with the cross-shaped flexible honeycombs filled in the trailing edge. Yin et al. [10] developed a variable camber wing with the pneumatic artificial muscle. Campanile et al. [11] studied aerodynamic deformations and aeroelastic amplification in the belt-rib concept proposed by German Aerospace Center. Smart materials [12-14] can be applied in the design of morphing structures, thus reducing the complexity due to the mechanical devices. Bae et al. [15] used piezoelectric laminate beam to establish

\footnotetext{
* Corresponding author: shiweizhao@buaa.edu.cn
} 
the morphing trailing edge. Barbarino et al. [16] investigated the effect of aerodynamic loading on a shape-memory-alloy-driven active-camber concept.

The design of morphing wings is the result of an optimization process based on multiple flight conditions. Bilgen et al. [17] presented a optimizer for a thin shell morphing airfoil driven by skin mounted microfiber composite actuators. Gaspari and Ricci [18] proposed a two-level optimization procedure for airfoil morphing design. Molinari et al. [19] proposed a multidisciplinary approach which optimizes aerodynamic and structural parameters of a morphing airfoil concurrently. This method has proved to be feasible and to yield superior performances compared to a sequential optimization. Thuwis et al. [20] presented an aero-servo-elastic optimization framework for a seamless morphing airfoil high-lift system.

Six bar drive mechanism is widely used in traditional mechanical structures such as sewing machine and pumping unit. In this paper, a morphing trailing edge based on the watt six-bar mechanism is proposed based on the multi-step optimization design method. In the first optimization stage, the most effective aerodynamic shape and bar position in the middle of the morphing trailing edge is determined. In the second optimization stage, the parameters of watt six link transmission mechanism are determined by genetic algorithm to match the optimal shape from the first optimization stage.

\section{Optimal design method}

\subsection{First optimization stage}

In the first optimization stage, the most effective aerodynamic shape and inner link position is determined. The most effective aerodynamic shape is obtained based on the aerodynamic analysis as shown in Fig.1. Therefore, the positions of bars in the middle of the morphing trailing edge is determined.

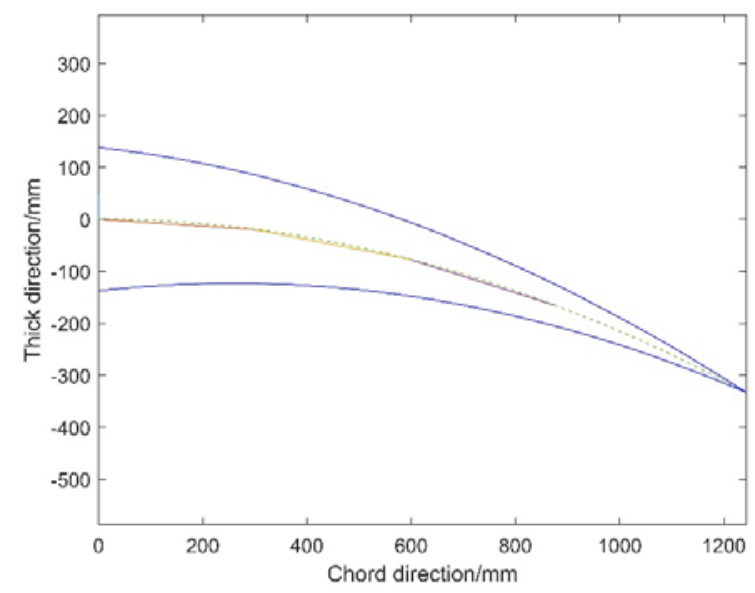

Fig. 1. Three bars in the middle of the morphing trailing edge.

\subsection{Second optimization stage}

In the second optimization stage, the parameters of watt six link transmission mechanism are determined by genetic algorithm to match the optimal shape from the first optimization stage. 
Original design of watt six-bar transmission mechanism for morphing trailing edge is shown in Fig.2. Deformation of original watt six-bar transmission mechanism is shown in Fig.3 which shows that the original design could not achieve the determined aerodynamic shape in the first optimization stage.



Fig. 2. Original design of watt six-bar transmission mechanism for morphing trailing edge.

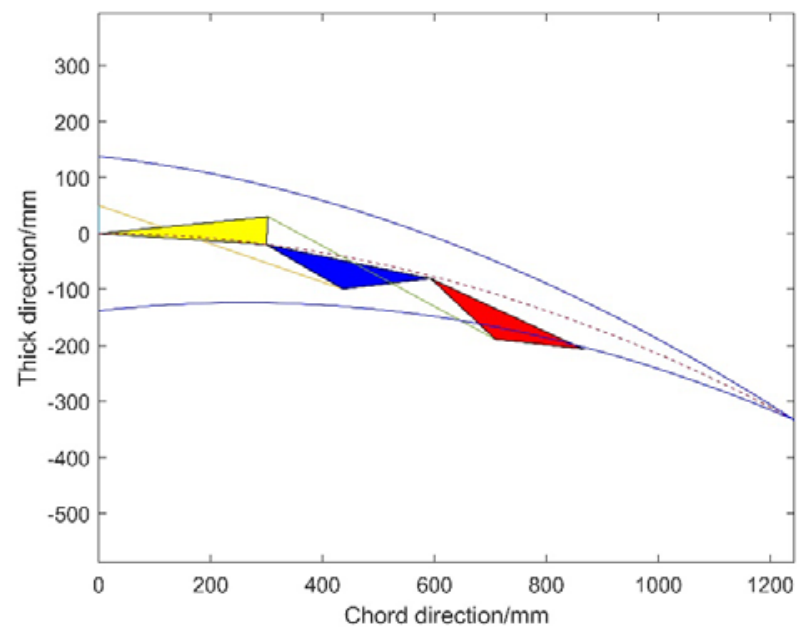

Fig. 3. Deformation of original watt six-bar transmission mechanism.

In order to achieve the determined aerodynamic shape in the first optimization stage, the original design is optimized based on the genetic algorithm. The objective is the sum of the position errors of the bar ends (point B, C and D) in the middle of the morphing trailing edge. The optimization parameters are the position of the point $\mathrm{E}, \mathrm{F}, \mathrm{G}$ and $\mathrm{H}$. The constraint is that all the points in the watt six-bar transmission mechanism are located inside the flexible trailing edge.

Genetic algorithm is used for optimization calculation. The population size is 200 . Maximum iteration generation is 400 . Fig.4 shows the optimal design of watt six-bar transmission mechanism for morphing trailing edge. Deformation of optimal watt six-bar transmission mechanism is shown in Fig.5 which shows that the optimal design could achieve the determined aerodynamic shape in the first optimization stage perfectly. 




Fig. 4. Optimal design of watt six-bar transmission mechanism for morphing trailing edge.

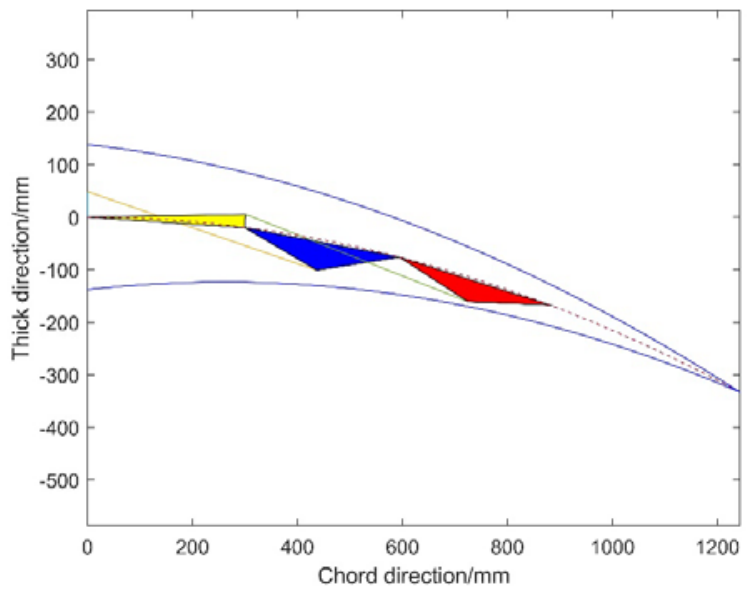

Fig. 5. Deformation of optimal watt six-bar transmission mechanism.

\section{Conclusion}

In this paper, a multi-step optimization design of watt six-bar transmission mechanism for morphing trailing edge is proposed. In the first optimization stage, the most effective aerodynamic shape and bar position in the middle of the morphing trailing edge is determined. In the second optimization stage, the parameters of watt six link transmission mechanism are determined by genetic algorithm to match the optimal shape from the first optimization stage. Result shows that the optimal design could achieve the determined aerodynamic shape in the first optimization stage perfectly.

The authors gratefully acknowledge the support from the National Natural Science Foundation of China under grant Nos. 11402014 and 11572023 and National Research Project "Variable camber wing technology(VCAN)". 


\section{Reference}

1. Li D, Zhao S, Da Ronch A, et al. A review of modelling and analysis of morphing wings. Progress in Aerospace Sciences, 2018, 100:46-62.

2. Woods BK, Bilgen O, Friswell MI. Wind tunnel testing of the fish bone active camber morphing concept. Journal of Intelligent Material Systems and Structures. 2014;25(7):772-85.

3. Chen Q, Bai P, Ying WL, Leng JS, Zhan HL, Liu ZQ. Analysis on the aerodynamic characteristics of variable camber airfoils with continuous smooth morphing trailing edge. Acta Aerodynamica Sinica. 2010;1:006.

4. Yokozeki T, Sugiura A, Hirano Y. Development of variable camber morphing airfoil using corrugated structure. Journal of Aircraft. 2014;51(3):1023-9.

5. Soneda K, Yokozeki T , Imamura T , et al. Aero-structural Analysis of Corrugated Morphing Wing with Spanwise Camber Change. AIAA Scitech 2020 Forum. 2020.

6. Woods BK, Dayyani I, Friswell MI. Fluid/structure-interaction analysis of the fish-bone-active-camber morphing concept. Journal of Aircraft. 2014;52(1):307-19.

7. Murugan S, Woods BK, Friswell MI. Hierarchical modeling and optimization of camber morphing airfoil. Aerospace Science and Technology. 2015;42:31-8.

8. Zhang J, Shaw A D , Wang C, et al. Aeroelastic model and analysis of an active camber morphing wing. Aerospace Science and Technology, 2021, 111(5):106534.

9. Zhang P, Zhou L, Cheng W, Qiu T. Conceptual design and experimental demonstration of a distributedly actuated morphing wing. Journal of Aircraft. 2014;52(2):452-61.

10. Yin W, Liu L, Chen Y, Leng J. Variable camber wing based on pneumatic artificial muscles. Second International Conference on Smart Materials and Nanotechnology in Engineering 2009. International Society for Optics and Photonics.

11. Campanile L F, Anders S. Aerodynamic and aeroelastic amplification in adaptive belt-rib airfoils. Aerospace Science \& Technology, 2005; 9(1):55-63.

12. A Y L , A S C , B T L , et al. Harnessing 3D printed residual stress to design heat-shrinkable metamaterials. Results in Physics, 2018, 11:85-95.

13. Stein $\mathrm{S}$, Wedler $\mathrm{J}$, Rhein $\mathrm{S}$, et al. A process chain for integrating piezoelectric transducers into aluminum die castings to generate smart lightweight structures. Results in Physics, 2017, 7:2534-2539.

14. Li X , Li Z , Huang H, et al. Broadband spring-connected bi-stable piezoelectric vibration energy harvester with variable potential barrier. Results in Physics, 2020, 18:103173.

15. Bae JS, Kyong NH, Seigler TM, Inman DJ. Aeroelastic considerations on shape control of an adaptive wing. Journal of intelligent material systems and structures. 2005;16(11-12):1051-6.

16. Barbarino S, Pecora R, Lecce L, et al. A Novel SMA-based Concept for Airfoil Structural Morphing. Journal of Materials Engineering \& Performance, 2009; 18(5-6):696-705.

17. Bilgen O, Flores E I S, Friswell M I. Optimization of Surface-Actuated Piezocomposite Variable-Camber Morphing Wings. ASME 2011 Conference on Smart Materials, Adaptive Structures and Intelligent Systems American Society of Mechanical Engineers, 2011:315-322. 
18. Gaspari A D, Ricci S. A Two-Level Approach for the Optimal Design of Morphing Wings Based On Compliant Structures. Journal of Intelligent Material Systems \& Structures, 2011; 22(10):1091-1111.

19. Molinari G, Quack M, Dmitriev V, et al. Aero-Structural Optimization of Morphing Airfoils for Adaptive Wings. Journal of Intelligent Material Systems \& Structures, 2011; 22(10):1075-1089.

20. Thuwis GA, Abdalla MM, Gürdal Z. Optimization of a variable-stiffness skin for morphing high-lift devices. Smart materials and structures. 2010;19(12):124010. 